CLINICAL STUDY

\title{
Circulating proinflammatory peptides related to abdominal adiposity and cardiometabolic risk factors in healthy prepubertal children
}

\author{
Sonya V Galcheva ${ }^{1}$, Violeta M Iotova ${ }^{1}$, Yoto $\mathrm{T} \mathrm{Yotov}^{2}$, Sergio Bernasconi ${ }^{3}$ and Maria E Street ${ }^{3}$ \\ Departments of ${ }^{1}$ Pediatrics and Medical Genetics and ${ }^{2}$ Internal Medicine, Varna Medical University, 55, Marin Drinov Street, Varna 9002 , Bulgaria and \\ ${ }^{3}$ Department of Pediatrics, University of Parma, Parma 43100, Italy
}

(Correspondence should be addressed to S V Galcheva; Email: sonya_galcheva@mail.bg)

\begin{abstract}
Objectives: To analyze the circulating levels of proinflammatory peptides in healthy prepubertal children in relation to abdominal obesity, measured by waist circumference (WC), and to investigate their interactions with cardiometabolic risk factors.

Design and methods: A cross-sectional study of 137 healthy prepubertal children with a mean age of $8.0 \pm 0.1$ years divided into three groups according to their $\mathrm{WC}$ as a measure of abdominal obesity: 'normal-WC' children (25th-75th percentile, $n=48$ ), 'children at risk' ( 75 th-90th percentile, $n=39$ ), and 'abdominally obese' ( $\geq 90$ th percentile, $n=50$ ) children. Auxological measurements and blood pressure (BP) were taken. Fasting levels of high-sensitivity C-reactive protein (hsCRP), interleukin 6 (IL6), tumor necrosis factor- $\alpha$ (TNF- $\alpha$ ), glucose, insulin, and lipid profile were measured. Insulin resistance (IR) was assessed by homeostasis model assessment of IR (HOMA-IR).

Results: Abdominally obese children had significantly higher BP, insulin, HOMA-IR, total cholesterol and triglycerides (TG) compared with their normal-WC counterparts $(P<0.05)$. HsCRP concentrations increased proportionally with the degree of abdominal obesity $(r=0.443, P<0.0001)$, whereas IL6 and TNF- $\alpha$ were not significantly associated with any of the adiposity variables. After controlling for adiposity, hsCRP was significantly correlated with systolic BP $(r=0.257, P=0.004)$, TNF- $\alpha$ levels were related to high-density lipoprotein cholesterol (HDL-C; $r=-0.216, P=0.016$ ) and TG $(r=0.196, P=0.029)$, whereas the relationship between IL6 and HDL-C reduced its magnitude to an insignificant level $(r=-0.173, P=0.055)$.

Conclusions: Healthy prepubertal children with abdominal obesity have associated inflammatory and cardiometabolic alterations, interacting with each other.

European Journal of Endocrinology $164553-558$
\end{abstract}

\section{Introduction}

Central fat distribution plays an important role in the development of obesity-related metabolic and cardiovascular abnormalities $(1,2)$. These disorders have been associated with the metabolic activity of visceral abdominal adipose tissue, an active endocrine organ, which secretes a large number of factors and cellular mediators, known as adipokines (3). Abdominal obesity has also been characterized by the presence of lowgrade chronic inflammation with an increased production of inflammatory peptides such as C-reactive protein (CRP), interleukin 6 (IL6), tumor necrosis factor- $\alpha$ (TNF- $\alpha$ ), plasminogen activator inhibitor-1, etc. (4). In adults, their serum concentrations have been reported to correlate positively with measures of adiposity (5) and to associate with the metabolic syndrome (MetS) (6), cardiovascular disease, insulin resistance (IR), and diabetes (7).
There is a growing interest in the function of the adiposity-related inflammatory factors and their contribution to the development of childhood MetS $(8,9)$. A number of studies have investigated the relationship between the circulating levels of adipokines, anthropometric indices and cardiometabolic risk factors in obese children and adolescents (10, 11). However, the majority of these have been limited mainly to associations between crude measures of adiposity such as body mass index (BMI) and CRP as a marker for an increased risk of coronary artery disease $(12,13)$, reporting age diversity of the participants $(14,15)$ or inconsistent results $(16,17)$. Less is known about the presence of low-grade systemic inflammation in healthy children with central obesity and on its potential associations with various cardiometabolic alterations before the onset of puberty $(18,19)$.

The aims of this study were i) to analyze the circulating levels of proinflammatory peptides (high-sensitivity CRP 
(hsCRP), IL6, and TNF- $\alpha$ ) in healthy prepubertal children in relation to abdominal obesity, measured by waist circumference (WC), and ii) to investigate the interactions of these proinflammatory peptides with cardiometabolic risk factors.

\section{Material and methods}

\section{Subjects}

A sample of 168 healthy prepubertal Caucasian children (78 boys and 90 girls), aged 6-10 years (mean age $8.1 \pm 1.2$ years), took part in this casecontrol study, conducted between December 2007 and May 2008. They were recruited from a representative cross-sectional study for the development of WC percentile curves for Bulgarian children and adolescents (20) through telephone or personal contact, representing $12 \%$ of the corresponding age group.

The exclusion criteria were adopted children with unknown family and past medical histories, initiated puberty, acute illness, chronic inflammatory diseases, other chronic diseases in general, or any medication intake at the time of investigation. The required information was obtained by a structured parental interview and full physical examination of the participants.

\section{Auxological and blood pressure measurements}

All anthropometric measurements were taken by the same well-trained investigator (S G). Weight was measured to the nearest $0.1 \mathrm{~kg}$ using a calibrated digital scale (TANITA Ltd, West Drayton, Middx, UK) with subjects standing barefoot in light clothing. Height was measured to the nearest $1 \mathrm{~mm}$ with a portable stadiometer (Seca Ltd, Hamburg, Germany), with the child upright and the head in the Frankfurt plane. WC was measured to the nearest $1 \mathrm{~mm}$ with a flexible, non-elastic tape midway between the tenth rib and the iliac crest at the end of a gentle expiration. BMI was calculated as weight $(\mathrm{kg}) /$ height $^{2}\left(\mathrm{~m}^{2}\right)$.

Pubertal development was clinically assessed based on secondary sexual characteristics according to Tanner's criteria (21). None of the subjects had begun puberty. Blood pressure (BP) was measured using a mercury sphygmomanometer according to the standard guidelines.

\section{Laboratory procedures and assays}

Venous blood samples were collected from all participants in a supine resting position after a 12-h overnight fast. Blood was centrifuged within $1 \mathrm{~h}$ and immediately assayed for glucose, total cholesterol (TC), triglycerides (TG), high-density lipoprotein cholesterol (HDL-C), and low-density lipoprotein cholesterol using enzymatic tests for the Olympus AU400 Clinical Chemistry system (Beckman Coulter, Inc., Brea, CA, USA). Serum was separated and kept frozen at $-20{ }^{\circ} \mathrm{C}$ until assayed for insulin, hsCRP, IL6, and TNF- $\alpha$. Serum insulin was determined by a sandwich ELISA with an assay sensitivity of $1.76 \mu \mathrm{IU} / \mathrm{ml}$ (DRG International, Inc., Mountainside, NJ, USA), and the intra- and inter-assay coefficients of variation were 2.6 and $2.88 \%$ respectively. The degree of IR was estimated using the homeostasis model assessment of IR (HOMA-IR) (22). For the analysis of hsCRP, a commercial ELISA kit was used (DRG International, Inc.) with a sensitivity of $0.1 \mathrm{mg} / \mathrm{l}$, and the intra- and inter-assay coefficients of variation were 2.3 and $2.5 \%$ respectively. IL6 and TNF$\alpha$ were evaluated by validated high-sensitive ELISAs (Quantikine HS, R\&D Systems, Minneapolis, MN, USA). The sensitivity of these assays was $0.016 \mathrm{pg} / \mathrm{ml}$ for IL6 and $0.038 \mathrm{pg} / \mathrm{ml}$ for TNF- $\alpha$. The intra- and inter-assay coefficients of variation were $<10 \%$ for both cytokines.

\section{Ethical approval}

The protocol was approved by the local ethical committee of Varna University Hospital, Bulgaria, and written parental informed consent and child's assent were taken prior to their enrollment into the study.

\section{Study design}

After a close inspection of the data for outliers, all analyses were performed excluding participants with hsCRP levels $>10 \mathrm{mg} / \mathrm{l}$ or those with missing data on different biochemical parameters $(n=31)$. This decision changed only the point estimates, but the direction of the effect did not differ from the analysis of the full sample. A total of 137 children (67 boys; mean age $8.0 \pm 0.1$ years) were included in the final analysis. They were divided into three groups based on the Bulgarian age- and sex-specific WC reference (20): 48 $(35.0 \%)$ were 'normal-WC' children (gr. 1) (25th to $<75$ th WC percentile), 39 (28.5\%) were "children at risk' (gr. 2) (75th to <90th WC percentile), and 50 (36.5\%) were 'abdominally obese' (gr. 3) (WC $\geq 90$ th percentile). No difference in age and sex ratio was observed between the study subgroups.

\section{Statistical analysis}

The results are presented as mean \pm s.D. or median (interquartile range). Each variable was tested for normal distribution, and log transformation was applied for BMI, insulin, HOMA-IR, TG, hsCRP, IL6, and TNF- $\alpha$ prior to further statistical testing. Gender differences were assessed using unpaired $t$-test. One-way ANOVA with Bonferroni's test for multiple comparisons was used to compare the circulating cytokines and the metabolic variables across the categories of abdominal adiposity, measured by WC. Partial Pearson's or Spearman's correlation coefficients with adjustment for age and sex were computed to examine the relationship between the variables. To judge the 
influence of adiposity on the outcome measures, ageand sex-adjusted partial correlation analyses were performed, using WC and BMI as additional confounding variables.

Several multiple linear regression models were applied, with backward or stepwise eliminations of the independent variables. The first set of regression equations was created to explore the linear relationship between the inflammatory markers as the dependent variables and the adiposity measures, age, and sex as the independent variables. The second set of regressions was created to look for the link between the different cardiometabolic parameters (TG, BP, insulin, HOMA-IR, etc.) as outcome variables and the inflammatory markers, i.e. hsCRP, IL6, and TNF- $\alpha$, as predictors, controlling for age, sex, BMI, and WC. The partial $t$-test statistic was used to determine the importance of each independent variable, with estimated $\beta$-coefficients. The $P$ values were set to be $<0.05$ to enter the model and $P>0.1$ to be removed from the model.

Statistical significance was set at a $P$ value $<0.05$. Statistical analysis was performed using SPSS software for Windows, version 15.0 (SPSS, Chicago, IL, USA).

\section{Results}

\section{Anthropometric indices and cardiometabolic features}

Children's clinical characteristics and biochemical parameters are presented in Table 1. Both WC and BMI increased significantly across the groups (from gr. 1 to gr. 3) in boys and girls $(P<0.0001)$, with a strong positive correlation between $\mathrm{WC}$ and BMI $(r=0.952$, $P<0.0001)$. Systolic and diastolic BP (SBP and DBP) increased significantly with the degree of abdominal obesity, measured by WC, without the presence of gender differences.

Although the markers of glucose homeostasis were within the normal range, serum insulin levels and HOMAIR increased significantly across the groups (Table 1). The abdominally obese children (gr. 3) had significantly higher levels of TC and TG compared with their normal-WC counterparts (gr. 1). No gender differences in serum lipids were detected $(P>0.05$ for all).

\section{Inflammatory peptides}

HsCRP concentrations showed a positive trend across the groups increasing proportionally with the degree of abdominal adiposity $(P<0.01$; Table 2$)$. Serum IL6 levels also increased with a significant difference found between normal-WC and abdominally obese children. TNF- $\alpha$ value did not differ among the WC percentile categories $(P>0.05)$. No gender-related differences were detected for hsCRP, IL6, and TNF- $\alpha$ levels $(P=0.29, P=0.163$, and $P=0.614$ respectively $)$.

\section{Correlations of inflammatory peptides with the measures of adiposity and cardiometabolic parameters}

HsCRP correlated with the measures of adiposity (Table 3). WC remained a significant predictor of hsCRP serum concentration even after an additional adjustment for BMI $(r=0.261, P=0.003)$.

HsCRP concentrations were significantly related to SBP, DBP, and HOMA index $(P<0.0001$ for all). This acute-phase protein correlated also with serum IL6 $(r=0.354, P<0.0001)$, but not with TNF- $\alpha$ levels $(r=0.02, P=0.825)$. The latter adipose tissue-derived cytokines showed a significant negative relationship

Table 1 Clinical characteristics and biochemical markers of prepubertal children by WC categories. Data are expressed as mean \pm S.D., median (interquartile range) or number of subjects.

\begin{tabular}{|c|c|c|c|c|}
\hline & Normal-WC gr. 1 & Children at risk gr. 2 & Abdominally obese gr. 3 & $P$ value \\
\hline Age (years) & $7.7 \pm 1.5$ & $8.1 \pm 1.0$ & $8.1 \pm 1.2$ & $\mathrm{NS}^{*,+, \neq}$ \\
\hline$n$ (male/female) & $22 / 26$ & $17 / 22$ & $28 / 22$ & $\mathrm{NS}^{*,+, \neq}$ \\
\hline Weight (kg) & $24.7 \pm 5.2$ & $35.4 \pm 5.2$ & $44.9 \pm 7.4$ & $<0.0001^{*, t, \ddagger}$ \\
\hline Height $(m)$ & $1.24 \pm 0.1$ & $1.33 \pm 0.07$ & $1.37 \pm 0.09$ & $<0.0001^{*, \dagger}$ \\
\hline BMI $\left(\mathrm{kg} / \mathrm{m}^{2}\right)$ & $18.1(17-19.8)$ & $23.8(22.3-25.7)$ & $28.5(27.3-30.6)$ & $<0.0001^{*, t, \mp}$ \\
\hline WC $(\mathrm{cm})$ & $54.7 \pm 3.9$ & $65.2 \pm 4.2$ & $76.4 \pm 5.3$ & $<0.0001^{*, t, \ddagger}$ \\
\hline $\mathrm{SBP}(\mathrm{mmHg})$ & $96 \pm \overline{8} .0$ & $108 \pm 8.7$ & $118.5 \pm 9.4$ & $<0.0001^{*, \dagger, \ddagger}$ \\
\hline $\mathrm{DBP}(\mathrm{mmHg})$ & $57.4 \pm 5.4$ & $65.7 \pm 4.5$ & $73.4 \pm 9.1$ & $<0.0001^{*, t, \ddagger}$ \\
\hline $\mathrm{TC}(\mathrm{mmol} / \mathrm{l})$ & $4.1 \pm 0.6$ & $4.2 \pm 0.7$ & $4.5 \pm 0.7$ & $0.012^{\dagger}$ \\
\hline $\mathrm{TG}(\mathrm{mmol} / \mathrm{l})$ & $0.61(0.51-0.96)$ & $0.69(0.55-0.83)$ & $0.94(0.68-1.26)$ & $<0.0001^{\dagger, \neq}$ \\
\hline $\mathrm{HDL}-\mathrm{C}(\mathrm{mmol} / \mathrm{l})$ & $1.3 \pm 0.3$ & $1.4 \pm 0.3$ & $1.2 \pm 0.3$ & $0.03^{\ddagger}$ \\
\hline LDL-C (mmol/l) & $2.5 \pm 0.4$ & $2.5 \pm 0.5$ & $2.8 \pm 0.7$ & $\mathrm{NS}^{*,+, \neq}$ \\
\hline Glucose (mmol/l) & $4.6 \pm 0.4$ & $4.8 \pm 0.5$ & $4.9 \pm 0.3$ & $0.015^{\dagger}$ \\
\hline Insulin $(\mu \mathrm{U} / \mathrm{ml})$ & $7.7(5.8-9.2)$ & $9.1(7.5-12)$ & $12.4(10.3-15.4)$ & $<0.01^{*, \dagger, \ddagger}$ \\
\hline HOMA-IR & $1.6(1.2-1.9)$ & $2.1(1.6-2.6)$ & $2.7(2.2-3.3)$ & $<0.01^{*, \dagger, \ddagger}$ \\
\hline
\end{tabular}

$P$ value: ${ }^{*}$ between normal-waist circumference and at risk group; ${ }^{\dagger}$ between normal-waist circumference and abdominally obese group; ${ }^{\ddagger}$ between at risk and abdominally obese group. NS, not significant; BMI, body mass index; WC, waist circumference; SBP, systolic blood pressure; DPB, diastolic blood pressure; TC, total cholesterol; TG, triglycerides; HDL-C, high-density lipoprotein cholesterol; LDL-C, low-density lipoprotein cholesterol; HOMA-IR, homeostasis model assessment of insulin resistance. 
Table 2 Inflammatory markers of prepubertal children subdivided by WC categories. Data are expressed as median (interquartile range).

\begin{tabular}{lcccc}
\hline & Normal-WC gr. 1 & Children at risk gr. 2 & Abdominally obese gr. 3 & $\boldsymbol{P}$ value \\
\hline HsCRP $(\mathrm{mg} / \mathrm{l})$ & $0.5(0.2-1.7)$ & $1.4(0.7-2.3)$ & $2.5(1.4-5.3)$ & $<0.01^{*,+, \neq}$ \\
IL6 $(\mathrm{pg} / \mathrm{ml})$ & $1.0(0.6-1.6)$ & $1.1(0.6-2.0)$ & $1.4(1.0-2.0)$ & $0.048^{\dagger}$ \\
TNF- $\alpha(\mathrm{pg} / \mathrm{ml})$ & $2.0(1.6-3.1)$ & $1.9(1.6-2.2)$ & $1.8(1.6-2.6)$ & $\mathrm{NS}$ \\
\hline
\end{tabular}

$P$ value: * between normal-waist circumference and at risk group; ${ }^{\dagger}$ between normal-waist circumference and abdominally obese group; ${ }^{\ddagger}$ between at risk and abdominally obese group. NS, not significant; WC, waist circumference; hsCRP, high-sensitivity C-reactive protein; IL6, interleukin-6; TNF- $\alpha$, tumor necrosis factor- $\alpha$.

with HDL-C values $(P=0.047$ and $P=0.028$ respectively), with no association with HOMA-IR or BP. Neither IL6 nor TNF- $\alpha$ were significantly associated with any of the adiposity variables.

After controlling for adiposity, hsCRP remained significantly positively associated with SBP $(r=0.257$, $P=0.004)$ and serum IL6 $(r=0.336, P<0.0001)$. TNF- $\alpha$ levels still correlated with HDL-C $(r=-0.216$, $P=0.016)$, TG $(r=0.196, P=0.029)$, and IL6 $(r=0.222, \quad P=0.013)$, whereas the relationship between IL6 and HDL-C reduced its magnitude to an insignificant level $(r=-0.173, P=0.055)$.

\section{Multiple regression analysis}

The first set of regression equations concerning the relationship of inflammatory peptides with measures of adiposity showed that hsCRP levels were significantly influenced by WC $(\beta=0.05, P<0.0001)$, with no significant effect of the latter on the cytokines' concentrations $(P>0.05)$. Regression analyses of the associations between cardiometabolic parameters, inflammatory proteins, and anthropometric measures revealed that in the entire group, WC contributed significantly to the levels of TG, insulin, HOMA-IR, and SBP values $\left(\beta_{\mathrm{TG}}=0.864, P=0.003 ; \beta_{\mathrm{ins}}=0.596\right.$, $P<0.0001 ; \beta_{\text {HOMA-IR }}=0.611, P<0.0001 ;$ and $\beta_{\mathrm{SBP}}=$ $0.440, P=0.022$ respectively). SBP was also influenced by hsCRP $(\beta=0.140, P=0.001)$, whereas BMI was a significant determinant for DBP $(\beta=0.757$, $P<0.0001)$. TNF- $\alpha$ had a predictive value only for HDL-C concentrations $(\beta=-0.185, P=0.035)$.

\section{Discussion}

This study is one of the few reports on the associations between central obesity, measured by WC and lowgrade inflammation in a non-patient group of healthy children, and on their contribution to the development of a cardiometabolic risk profile even before the onset of puberty. The lack of adult confounding factors (smoking, advanced vascular disease, chronic conditions, etc.) in our young participants confers a certain advantage in clarifying these interrelationships.

While most studies examining obesity and inflammatory factors in children used BMI as a measure of adiposity, we investigated the proposed associations assessing the influence of abdominal fat mass, measured by WC. This decision was related to the fact that central adiposity has been more strongly associated with adult cardiovascular disease and diabetes risk even in subjects who are lean or simply overweight by BMI $(6,23)$.

The presence of low-grade inflammation in the study group was assessed by measuring serum concentrations of hsCRP, IL6, and TNF- $\alpha$. HsCRP is an acute-phase protein, produced exclusively by the liver in response to inflammatory cytokines (24). Published evidence associates it with the development of cardiovascular events considering it as an important determinant of atherosclerotic vascular changes even in young obese children $(25,26)$. In our study, we found higher concentrations of this inflammatory protein in abdominally obese healthy children compared with their normal-WC counterparts even before the onset of puberty. Furthermore, it correlated significantly with the measure of central fat distribution, as waist girth was the only anthropometric predictor determining its increase. It is worth mentioning that most of the studies reporting similar findings have used population-based samples of children and adolescents of a greater age range $(10,12)$ or entirely clinically based study groups (25). Moreover, some authors investigated the relationships with the overall adiposity measures $(25,27)$ or measured non-sensitive CRP (28).

We showed the presence of a positive correlation between the increased hsCRP and several cardiometabolic risk factors. This was consistent with other studies

Table 3 Partial correlations between inflammatory markers and adiposity or cardiometabolic measures in prepubertal children, controlled for age and sex.

\begin{tabular}{lllllrrrrr}
\hline & WC & BMI & SBP & DBP & Glucose & Insulin & HOMA-IR & TG & HDL-C \\
\hline HsCRP & $0.443^{\ddagger}$ & $0.389^{\ddagger}$ & $0.474^{\ddagger}$ & $0.375^{\ddagger}$ & 0.126 & $0.278^{\ddagger}$ & $0.285^{\ddagger}$ & 0.119 & -0.141 \\
IL6 & 0.150 & 0.163 & 0.165 & 0.099 & -0.047 & 0.098 & 0.082 & 0.013 & $-0.177^{\star}$ \\
TNF- $\alpha$ & -0.146 & -0.147 & -0.146 & -0.086 & -0.127 & -0.154 & -0.168 & 0.141 & $-0.196^{\star}$ \\
\hline
\end{tabular}

${ }^{\star} P<0.05$ and ${ }^{\ddagger} P<0.0001$. WC, waist circumference; BMI, body mass index; SBP, systolic blood pressure; DPB, diastolic blood pressure; HOMA-IR, homeostasis model assessment of insulin resistance; TG, triglycerides; HDL-C, high-density lipoprotein cholesterol. 
linking MetS components to hsCRP levels in children and adolescents $(8,13,26)$. However, similar to other reports (29), the associations (except for SBP) disappeared after controlling for adiposity measures, suggesting that in healthy prepubertal children, the observed relationships were mediated through the obesity status. Hence, it could be hypothesized that a longer period of exposure to or accumulation of excessive fat mass may be required, or a critical degree of inflammation must be reached before the effects of hsCRP become significant. The independent positive correlation between hsCRP and SBP in our study population could be connected with the inflammatoryinduced vascular changes leading to an increased risk of developing a hypertensive condition in early childhood (30).

The other two investigated markers of low-grade inflammation, IL6 and TNF- $\alpha$, play a central stimulatory role in the production of the acute-phase proteins and correlate with the adiposity indices and central fat distribution as well as with the development of IR and MetS in adults (4).

Previous studies, investigating the links between these cytokines, obesity, and cardiometabolic abnormalities in children, have shown inconsistent results. In contrast to a few reports conducted in Caucasian cohorts $(15,19)$ and despite the higher IL6 concentrations detected in the abdominally obese children, we found no positive association between its levels and the adiposity measures or cardiometabolic risk factors. TNF- $\alpha$ was also unrelated to the abdominal obesity of our participants and had an independent predictive value only for the decreased concentrations of HDL-C. Similar data in children were demonstrated by Steene-Johannessen et al. (10) and others $(26,31)$, while Caballero et al. (11) and Aygun et al. (19) reported raised TNF- $\alpha$ in obese children.

Some potential reasons for these discrepancies could be the differences in age, race, pubertal stage, obesity degree, and fat distribution among different studies. Our results do not rule out IL6 and TNF- $\alpha$ as potential contributors for future cardiometabolic risks. We suppose that the relatively low overall and abdominal obesity grade among the participants explain the moderate elevation of the studied cytokine levels.

Adult studies have demonstrated sex dimorphism in adipose tissue metabolism and inflammation that could be explained by the well-documented gender differences in adult body fat distribution and estrogen effects on cytokine regulation and production of inflammatory proteins by the liver $(32,33)$. IL6 and TNF- $\alpha$ probably become important players at puberty and postpuberty, when their activities are associated with those of other inflammatory cytokines as IL1 $\beta$, IL8, etc. (3). This hypothesis has partially been proven by a longitudinal study of 118 healthy children, which found raised IL6 levels in 15-year-old overweight/obese girls with no difference at the age of 8 years (34). In our prepubertal group, the concentrations of all investigated inflammatory markers did not show significant gender differences and thus confirmed other international reports in children $(15,18,25)$.

The main strengths of this study include i) the recruitment of healthy prepubertal participants before the development of adult chronic diseases, strictly controlling for possible biases and ii) the investigation of a number of inflammatory and cardiometabolic parameters, known to be closely associated with abdominal adiposity in adults.

The limitation of the study is its cross-sectional design, which makes it impossible to determine the causal interactions between the inflammatory and cardiometabolic factors and does not allow examining longitudinal consequences of the abdominal adiposity.

In conclusion, abdominal obesity, measured by WC, is associated with inflammatory and cardiometabolic alterations in apparently healthy prepubertal children, and these alterations interact with each other. These changes could persist into adulthood representing an increased risk for development of cardiovascular disease and diabetes. Therefore, further research into the field of abdominal obesity-related inflammation is required for those children who are at risk of excessive central fat accumulation even before the onset of puberty.

\section{Declaration of interest}

The authors declare that there is no conflict of interest that could be perceived as prejudicing the impartiality of the research reported.

\section{Funding}

This study was supported by an ESPE Research Fellowship sponsored by Novo Nordisk and by a grant of 'Medical Science Fund' of Varna Medical University, Varna, Bulgaria.

\section{Acknowledgements}

The authors would like to thank all the children and their parents who took part in the study. We are also grateful to Dr Emil Dyankov (University Hospital, Varna, Bulgaria) for the data collection, Maria A Ziveri (University of Parma, Parma, Italy) for the analysis of the proinflammatory cytokines, and Drs Tancheva and Toshkina (Bulgaria) for the rest of the laboratory analyses.

\section{References}

1 Ritchie SA \& Connell JMC. The link between abdominal obesity, metabolic syndrome and cardiovascular disease. Nutrition, Metabolism, and Cardiovascular Diseases $2007 \quad 17$ 319-326. (doi:10.1016/j.numecd.2006.07.005)

2 McCarthy HD. Body fat measurements in children as predictors for the metabolic syndrome: focus on waist circumference. Proceedings of the Nutrition Society 200665 385-392. (doi:10.1079/PNS2006514)

3 Trayhurn P \& Wood IS. Adipokines: inflammation and the pleiotropic role of white adipose tissue. British Journal of Nutrition 200492 347-355. (doi:10.1079/BJN20041213)

4 Hotamisligil GS. Inflammation and metabolic disorders. Nature 2006444 860-867. (doi:10.1038/nature05485)

5 Park HS, Park JY \& Yu R. Relationship of obesity and visceral adiposity with serum concentrations of CRP, TNF-alpha and IL-6. Diabetes Research and Clinical Practice 200569 29-35. (doi:10. 1016/j.diabres.2004.11.007) 
6 Stenholm S, Koster A, Alley DE, Visser M, Maggio M, Harris TB, Egan JM, Bandinelli S, Guralnik JM \& Ferucci L. Adipocytokines and the metabolic syndrome among older persons with and without obesity - the InCHIANTI Study. Clinical Endocrinology 201073 55-65. (doi:10.1111/j.1365-2265.2009.03742.x)

7 de Rooij SR, Nilsson PM, Nolan JJ, Gabriel R, Bobbioni-Harsch E, Mingrone G, Dekker JM \& Relationship Between Insulin Sensitivity and Cardiovascular Disease (RISC) Investigators. Low-grade chronic inflammation in the relationship between insulin sensitivity and cardiovascular disease (RISC) population: associations with insulin resistance and cardiometabolic risk profile. Diabetes Care 200932 1295-1301. (doi:10.2337/dc08-1795)

8 Warnberg J \& Marcos A. Low-grade inflammation and the metabolic syndrome in children and adolescents. Current Opinion in Lipidology 200819 11-15. (doi:10.1097/MOL.0b013e3282f4096b)

9 Schwarzenberg SJ \& Sinaiko AR. Obesity and inflammation in children. Paediatric Respiratory Reviews 20067 239-246. (doi:10. 1016/j.prrv.2006.08.002)

10 Steene-Johannessen J, Kolle E, Eseland EJ, Anderssen SA \& Anderssen LB. Waist circumference is related to low-grade inflammation in youth. International Journal of Pediatric Obesity 20105 313-319. (doi:10.3109/17477160903497035)

11 Caballero AE, Bousquet-Santos K, Robles-Osorio L, Montagnani V, Soodini G, Porramatikul S, Hamdy O, Nobrega AC \& Horton ES. Overweight Latino children and adolescents have marked endothelial dysfunction and subclinical vascular inflammation in association with excess body fat and insulin resistance. Diabetes Care 200831 576-582. (doi:10.2337/dc07-1540)

12 Ford ES, Galuska DA, Gillespie C, Will JC, Giles WH \& Dietz WH. C-reactive protein and body mass index in children: findings from the third National Health and Nutrition Examination Survey, 1988-1994. Journal of Pediatrics 2001138 486-492. (doi:10. 1067/mpd.2001.112898)

13 Soriano-Guillen L, Hernandez-Garcia B, Pita J, DominguezGarrido N, Del Rio-Camacho G \& Rovira A. High-sensitivity C-reactive protein is a good marker of cardiovascular risk in obese children and adolescents. European Journal of Endocrinology 2008 159 R1-R4. (doi:10.1530/EJE-08-0212)

14 Rubin DA, McMurray RG, Harrell JS, Hackney AC, Thorpe DE \& Haqq AM. The association between insulin resistance and cytokines in adolescents: the role of weight status and exercise. Metabolism 200857 683-690. (doi:10.1016/j.metabol.2008.01.005)

15 Mauras N, DelGiorno C, Kollman C, Bird K, Morgan M, Sweeten S, Balagopal P \& Damaso L. Obesity without established comorbidities of the metabolic syndrome is associated with a proinflammatory and prothrombotic state, even before the onset of puberty in children. Journal of Clinical Endocrinology and Metabolism 201095 1060-1068. (doi:10.1210/jc.2009-1887)

16 Wärnberg J, Nova E, Moreno LA, Romeo J, Mesana MI, Ruiz JR, Ortega FB, Sjöström M, Bueno M, Marcos A \& AVENA Study Group. Inflammatory proteins are related to total and abdominal adiposity in a healthy adolescent population: the AVENA Study. American Journal of Clinical Nutrition 200684 505-512.

17 Alvarez JA, Higgins PB, Oster RA. Fernandez JR, Darnell BE \& Gower BA. Fasting and postprandial markers of inflammation in lean and overweight children. American Journal of Clinical Nutrition 200989 1138-1144. (doi:10.3945/ajcn.2008.26926)

18 Valle M, Martos R, Gascon F, Canete R, Zafra MA \& Morales R. Lowgrade systemic inflammation, hypoadiponectinemia and a high concentration of leptin are present in very young obese children, and correlate with metabolic syndrome. Diabetes and Metabolism 2005 31 55-62. (doi:10.1016/S1262-3636(07)70167-2)

19 Aygun AD, Gungor S, Ustundag B, Gurgoze MK \& Sen Y. Proinflammatory cytokines and leptin are increased in serum of prepubertal obese children. Mediators of Inflammation 20053 180-183. (doi:10.1155/MI.2005.180)

20 Galcheva SV, Iotova VM, Yotov YT, Grozdeva KP, Stratev VK \& Tzaneva VI. Waist circumference percentile curves for Bulgarian children and adolescents aged 6-18 years. International Journal of Pediatric Obesity 20094 381-388. (doi:10.3109/ 17477160902846195)

21 Tanner JM, Whitehouse RH \& Takaishi M. Standards for birth to maturity for height, weight, height and weight velocity: British children. Archives of Disease in Childhood 196541 454-495. (doi:10.1136/adc.41.219.454)

22 Mathews D, Hosker J, Rudenski A, Naylor B, Treacher D \& Turner R. Homeostasis model assessment: insulin resistance and beta-cell function from fasting plasma glucose and insulin concentration in man. Diabetologia 198528 412-419. (doi:10. 1007/BF00280883)

23 Balkau B, Deanfield JE, Despres JP, Bassand JP, Fox KA, Smith SC, Barter P, Tan CE, Van Gaal L, Wittchen HU, Massien C \& Haffner SM. International day for the evaluation of abdominal obesity (IDEA): a study of waist circumference, cardiovascular disease and diabetes mellitus in 168,000 primary care patients in 63 countries. Circulation 2007116 1942-1951. (doi:10.1161/ CIRCULATIONAHA.106.676379)

24 Yeh ET. A new perspective on the biology of C-reactive protein. Circulation Research 200597 609-611. (doi:10.1161/01.RES. $0000186188.38344 .13)$

25 Kapiotis S, Holzer G, Schaller G, Haumer M, Widhalm H, Weghuber D, Jilma B, Rogla G, Woltz M, Widhalm K \& Wagner OF. A proinflammatory state is detectable in obese children and is accompanied by functional and morphological vascular changes. Arteriosclerosis, Thrombosis, and Vascular Biology 200626 2541-2546. (doi:10.1161/01.ATV.0000245795.08139.70)

26 Valle JM, Estepa RM, Camacho RM, Estrada RC, Luna FG \& Guitarte FB. Endothelial dysfunction is related to insulin resistance and inflammatory biomarker levels in obese prepubertal children. European Journal of Endocrinology 2007156 497-502. (doi:10. 1530/EJE-06-0662)

27 Skinner AC, Steiner MJ, Henderson FW \& Perrin EM. Multiple markers of inflammation and weight status: cross-sectional analyses throughout childhood. Pediatrics $2010 \mathbf{1 2 5}$ e801-e809. (doi:10.1542/peds.2009-2182)

28 Gillum RF. Association of serum C-reactive protein and indices of body fat distribution and overweight in Mexican American children. Journal of the National Medical Association 200395 545-552.

29 Moran A, Steffen LM, Jacobs DR, Steinberger J, Pankow JS, Hong CP, Tracy RP \& Sinaiko AR. Relation of C-reactive protein to insulin resistance and cardiovascular risk factors in youth. Diabetes Care 200528 1763-1768. (doi:10.2337/diacare.28.7.1763)

30 Lande MB, Pearson TA, Vermilion RP, Auinger P \& Fernandez ID. Elevated blood pressure, race/ethnicity, and C-reactive protein levels in children and adolescents. Pediatrics $2008 \mathbf{1 2 2}$ 1252-1257. (doi:10.1542/peds.2007-3162)

31 McFarlin BK, Jonston CA, Tyler C, Hutchison AT, Kuent ML, Reeves R \& Foreyt JP. Inflammatory markers are elevated in overweight Mexican-American children. International Journal of Pediatric Obesity 20072 235-241. (doi:10.1080/17477160701440455)

32 Lakoski SG, Cushman M, Criqui M, Rundek T, Blumenthal RS, D'Agostino RB Jr \& Herrington DM. Gender and C-reactive protein: data from the Multiethnic Study of Atherosclerosis (MESA) cohort. American Heart Journal 2006152 593-598. (doi:10.1016/j.ahj. 2006.02.015)

33 Cartier A, Cote M, Lemieux I, Perusse L, Tremblay A, Bouchard C \& Depres JP. Sex differences in inflammatory markers: what is the contribution of visceral adiposity? American Journal of Clinical Nutrition 200989 1307-1314. (doi:10.3945/ajen.2008.27030)

34 Tam CS, Garnett SP, Cowell CT, Heilbronn LK, Lee JW, Wong M \& Baur LA. IL-6, IL-8 and IL-10 levels in healthy weight and overweight children. Hormone Research in Pediatrics 201073 128-134. (doi:10.1159/000277632)

Received 1 December 2010

Accepted 11 January 2011 